\title{
Frontal brain asymmetries as effective parameters to assess the quality of audiovisual stimuli perception in adult and young cochlear implant users
}

\author{
Asimmetria nell'attività cerebrale frontale come parametro efficace della qualità \\ percettiva degli stimoli audiovisivi in portatori di impianto cocleare giovani e adulti

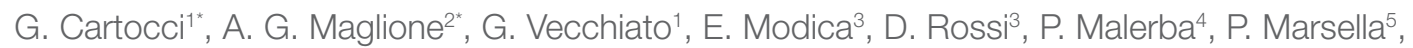 \\ A. Scorpecci ${ }^{5}$, S. Giannantonio ${ }^{5}$, F. Mosca ${ }^{6}$, C. A. Leone ${ }^{6}$, R. Grassia ${ }^{6}$, F. Babiloni ${ }^{1,2}$ \\ ${ }^{1}$ Department of Molecular Medicine, Sapienza University of Rome, Italy; ${ }^{2}$ BrainSigns Srl, Rome, Italy; ${ }^{3}$ Department \\ of Anatomical, Histological, Forensic \& Orthopedic Sciences, Sapienza University of Rome, Italy; ${ }^{4}$ Cochlear Italia \\ Srl., Bologna, Italy; ${ }^{5}$ Department of Otorhinolaryngology, Audiology and Otology Unit, "Bambino Gesù" Pediatric \\ Hospital, Rome, Italy; ${ }^{6}$ ENT Department, Azienda Ospedaliera Dei Colli Monaldi, Naples, Italy \\ These authors equally contributed to the present article.
}

\begin{abstract}
SUMMARY
How is music perceived by cochlear implant (CI) users? This question arises as "the next step" given the impressive performance obtained by these patients in language perception. Furthermore, how can music perception be evaluated beyond self-report rating, in order to obtain measurable data? To address this question, estimation of the frontal electroencephalographic (EEG) alpha activity imbalance, acquired through a 19-channel EEG cap, appears to be a suitable instrument to measure the approach/withdrawal (AW index) reaction to external stimuli. Specifically, a greater value of AW indicates an increased propensity to stimulus approach, and vice versa a lower one a tendency to withdraw from the stimulus. Additionally, due to prelingually and postlingually deafened pathology acquisition, children and adults, respectively, would probably differ in music perception. The aim of the present study was to investigate children and adult CI users, in unilateral (UCI) and bilateral (BCI) implantation conditions, during three experimental situations of music exposure (normal, distorted and mute). Additionally, a study of functional connectivity patterns within cerebral networks was performed to investigate functioning patterns in different experimental populations. As a general result, congruency among patterns between BCI patients and control (CTRL) subjects was seen, characterised by lowest values for the distorted condition (vs. normal and mute conditions) in the AW index and in the connectivity analysis. Additionally, the normal and distorted conditions were significantly different in CI and CTRL adults, and in CTRL children, but not in CI children. These results suggest a higher capacity of discrimination and approach motivation towards normal music in CTRL and BCI subjects, but not for UCI patients. Therefore, for perception of music CTRL and BCI participants appear more similar than UCI subjects, as estimated by measurable and not self-reported parameters.
\end{abstract}

KEY WORDS: Approach/Withdrawal • Alpha rhythm • Electroencephalography • Music • Functional connectivity $\bullet$ Graph Theory

\section{RIASSUNTO}

Come è percepita la musica dai portatori di impianto cocleare (CI)? Questa domanda sorge come la "prossima sfida", date le impressionanti prestazioni ottenute da questi pazienti nella percezione del linguaggio. Inoltre, come valutare la percezione della musica oltre il dichiarato verbale, così da ottenere dati misurabili? Per rispondere a tale domanda la stima dell'asimmetria dell'attività elettroencefalografica (EEG) in banda alfa, acquisita tramite una cuffia a 19 canali, risulta un mezzo adatto a misurare la tendenza all'approccio o al rifiuto (indice AW) verso uno stimolo. Specificamente, un maggior valore di AW indica una maggiore propensione all'approccio, viceversa un minor valore di $A W$ una tendenza ad evitare un determinato stimolo. Inoltre, a causa dell'acquisizione prelinguale e postlinguale della sordità, bambini ed adulti rispettivamente potrebbero differire nella percezione della musica. Scopo del presente studio è stato quello di indagare due popolazioni di portatori di impianto cocleare di diversa età, bambini e adulti, nelle condizioni di impianto cocleare unilaterale (UCI) e impianto cocleare bilaterale (BCI), durante l'esposizione a tre condizioni sperimentali di stimolo musicale (Normale, Distorto e Muto). Inoltre, è stato effettuato uno studio di modelli di connettività funzionale tra reti cerebrali, così da investigare eventuali pattern funzionali peculiari delle diverse popolazioni. Come risultato generale, sia negli adulti che nei bambini, è stato dimostrata una congruenza tra i pattern elettroencefalografici riportati in pazienti BCI e soggetti di controllo normoudenti (CTRL), caratterizzata da valori più bassi per la condizione Distorto (rispetto alle condizioni Normale e Muto) nell'indice AW e nell'analisi di connettività. Inoltre, la condizione Normale e Distorta risultavano differenti in modo statisticamente significativo per il gruppo degli adulti con impianto cocleare e nei CTRL, così come nel gruppo bambini CTRL, ma non nei bambini con impianto cocleare. Queste evidenze suggeriscono una maggiore capacità di discriminazione e di motivazione all'approccio verso la musica Normale per i soggetti CTRL e BCI, a causa della somiglianza nella percezione della musica per questi due gruppi, in particolare per gli adulti, ma non per i pazienti UCI. Di conseguenza soggetti CTRL e BCI appaiono più simili che gli UCI nella percezione della musica Normale, come stimato da parametri misurabili, non derivanti da dati auto-dichiarati dai pazienti. 


\section{Introduction}

Musical sounds, since prehistory, have always exerted rewarding and emotional effects on humans ${ }^{1}$, and research on music perception and its effects have seen a large rise in recent years ${ }^{2}$. In this field, cochlear implant recipients are receiving growing interest, particularly in relation to the study of patients' quality of life ${ }^{3}$, evaluation of bilateral implantation in adults as a good clinical practice ${ }^{4}$ and possible development of specific training for patients ${ }^{56}$. Concerning this latter point, an intriguing example has been shown in a recent electroencephalographic (EEG) event related potentials study, where singing was associated with increased development of neural networks for attention and more accurate neural discrimination in cochlear implanted (CI) children ${ }^{7}$. Additionally, musically trained deaf children (hearing aid and/or CI users) showed better performance in auditory scene analysis, auditory working memory and phonetic discrimination tasks in comparison to untrained peers, and the better performances on these tasks might at least be partly driven by music lessons ${ }^{8}$.

It is well-known from literature that $\mathrm{CI}$ users experience difficulties in perceiving music. This is probably due to the constraint of the CI in transmission of the spectral information of music, and to the complexity of pitch relationships between notes, both at the basis of the perception of the melody ${ }^{9-12}$. Several neuroimaging studies investigating the cortical activation of CI users during speech-language perception showed an increased activation of already present auditory networks, i.e. brain areas traditionally employed for auditory processing; in addition, CI users demonstrate plastic reorganisation of normally occurring networks, including recruitment of brain areas not traditionally utilised for auditory processing ${ }^{9}$. Furthermore, studies on experienced CI users suggest that the degree of activation (in terms of both extent and intensity) of auditory cortex in response to speech stimuli corresponds to the degree of success in speech perception ${ }^{10}$. In fact, even if language perception has reached impressive levels in this population, also characterised by high auditory cortical activation ${ }^{14}$, music perception still remains challenging. In fact, intriguing evidence of higher and lower cortical activity for rhythm and melody perception has been found ${ }^{14}$. Therefore, the study of cortical activation in response to music exposure in CI users appears worthy, since it has been repeatedly shown altered cortical activity in CI subjects in comparison to normal hearing ones. Additionally, due to prelingually and postlingually deafened pathology acquisition, which frequently depicts the condition in children and adults, respectively, they would probably result in a different music perception.
This statement is suggested by studies showing peculiar frontal cortex activity in prelingual and postlingual deafness. For instance, it has been shown a larger mismatch negativity, generated by the frontal cortex, in CI prelingually deafened patients, related to better speech performances ${ }^{15}$. In addition, CI postlingually deafened patients showed increased cortical activity in comparison to normal hearing subjects, probably due to the use of already existing speech sound networks ${ }^{16}$.

This hypothesis is also indirectly suggested by the evidence that later cochlear implantation is correlated with an improved hearing cues in music ability as tested by the Montreal Battery of Evaluation of Amusia (MBEA) in unilateral CI (UCI) children, probably due to the low frequency access in older implanted patients ${ }^{17}$. Evidence also suggests poorer music perception in CI children in comparison to adults ${ }^{18}$. Instead, with respect to normal hearing $(\mathrm{NH})$ peers, UCI children were less accurate but best able to discern rhythm changes and to remember musical pieces ${ }^{17}$. Concerning music perception and the relative induced pleasantness, the available data have shown that both children and young adults CI recipients succeed in identifying the original and instrumental versions of familiar recorded songs. Although favourably evaluated, they were not able to identify different melody versions ${ }^{19}$.

In addition, the study of cerebral activations in CI recipients may provide additional perspectives to investigate the possible benefits of surgery and musical therapies. In particular, motivation and affective processes could play an important role for a better comprehension of musical messages. In this regard, the prefrontal cortex (PFC) appears to play a pivotal role in a larger overall circuit involved in emotional and motivational processes ${ }^{20}$. In fact, frontal EEG alpha activity is frequently used to detect intra- and between-subject asymmetries in cortical activation. According to the "withdrawal/approach" model, analyses of the EEG power spectrum suggest a different lateralisation for the anterior cerebral hemispheres in approach and withdrawal motivational tendencies and emotions ${ }^{21}$. Specifically, a relative power suppression of the alpha rhythm across the left PFC is associated with a propensity to engage toward a stimulus. The application of EEG withdrawal/approach analysis to the reaction to music exposure has been investigated, highlighting a correlation between the reported valence (pleasantness/ unpleasantness) and arousal (intensity/energy) of musical stimuli with frontal alpha $(8-13 \mathrm{~Hz})$ asymmetry ${ }^{22}$. In particular, these authors revealed a greater relative left frontal EEG activity to joy and happy musical pieces and greater relative right frontal EEG activity to fear and sad ones. 
Experimental evidence in adult CI users in line with this theory have been already provided. The approach towards musical video clips, indexed by the frontal EEG alpha activity imbalance, in a $\mathrm{NH}$ population and in bilateral CI (BCI) users presented similar range of variation across different stimuli conditions (normal, distorted and mute), in particular with a total excursion of 0.5 and $0.55 \mathrm{z}$-score units of the alpha frontal asymmetry index among the conditions; UCI patients reported a higher range of variation in comparison to the other groups, presenting a total excursion of $3.5 \mathrm{z}$-score units ${ }^{23}$.

EEG alpha asymmetries have also been investigated through estimation of the cortical sources of prelingually deaf implanted children during music exposure. In particular, the inconsistency with the approach/withdrawal model in comparison to NH subjects was seen in the UCI condition, suggesting an impairment in discriminating normal from dissonant music and appreciating the pleasantness of normal music ${ }^{24}$. A preliminary study suggested less approach and interest in music for one UCI user with respect to one $\mathrm{BCI}$ user and one $\mathrm{NH}$ child ${ }^{25}$. Additionally, the pattern of the EEG power spectrum in the alpha band in UCI children indicated more approach (as reflected by the higher alpha synchronization) for mute and distorted music listening than for the Normal audio condition, while $\mathrm{NH}$ children and $\mathrm{BCI}$ users showed cortical activation that was more similar among the two groups, characterised by higher approach for normal audio in comparison to both mute and distorted audio conditions. Such findings suggested a closer to normal music perception for the BCI users ${ }^{26}$.

Moreover, it appears extremely interesting and informative to extend such investigations to different technical approaches. A relation between cerebral rhythms and connectivity has been suggested in several articles, and synchronisation in different frequency bands may correspond to different networks and different cognitive functions ${ }^{27}$, also enabling the discrimination between healthy and pathological brain functioning ${ }^{28}$.

The brain is a complex system where spatially segregated areas are interconnected. Starting from this perspective, brain network properties can be represented by functional connectivity patterns, and graph theory provides useful quantitative indices to measure these patterns ${ }^{29}$. Previous findings in $\mathrm{NH}$ subjects provided evidence for an increase of the number of functional connections and a more random network structure in a portion of the alpha band during music perception, in comparison to both noise and silence conditions. This result supports the hypothesis of a positive effect (higher efficiency) of music on human brain functional networks ${ }^{30}$. Furthermore, a principal compo- nent analysis study showed asymmetry in the pre-frontal cortex relating to a number of emotions induced by different kinds of music (pleasantness, energy, tension, anger, fear, happiness, sadness and tenderness ${ }^{31}$. Despite its importance in understanding physiological and pathophysiological conditions, functional connectivity estimation has not been applied to study music perception in CI users. In the light of the aforementioned background, the objective of the present study was twofold. Firstly, because of the differences due to the unilateral and bilateral amplification and to the prelingually and postlingually deafened acquisition, we investigated two age populations in unilateral and bilateral cochlear implantation conditions, children and adult implant users, during three experimental situations of music exposure. Secondly, given the absence of published data concerning the functional connectivity during music exposure in these populations, we also characterised connectivity patterns in the same musical experimental conditions (normal, distorted and mute).

Towards these aims, two age populations, children and adults, were recruited according to three experimental groups (BCI, UCI and NH participants), who underwent EEG recording during exposition to three conditions (normal, distorted and mute) of a movie with explicit musical content. The frontal brain asymmetry in the alpha band activity was calculated to quantify the approach expressed by subjects in the three experimental conditions. Furthermore, brain asymmetry calculation was also performed via graph theory on the functional brain connectivity patterns investigated in the same testing conditions.

To our knowledge, this is the first study on music perception in cochlear implanted patients that also investigates functional brain connectivity.

\section{Materials and methods}

\section{Experimental design with paediatric subjects}

The paediatric population was composed of $6 \mathrm{NH}$ control subjects, 7 UCI and 4 BCI patients (Tables I and II). All children in the study were affected by bilateral, profound, sensorineural hearing loss. None had usable residual hearing (i.e. auditory threshold better than $100 \mathrm{~dB}$ HL) in either ear before cochlear implantation. In the unilateral CI condition, patients had no usable residual hearing in the non-implanted ear, and were poor contralateral hearing aid users. All patients included in the study had been receiving auditory-verbal therapy for a minimum of three years. With unilateral CI, they achieved a $20 \mathrm{~dB}$ HL PTA for all tested pure tone frequencies. By the time they were included in the study, they had good speech comprehension skills, i.e. $100 \%$ score obtained in quiet (assessed 
Table I. Paediatric groups age and time of cochlear implant experience.

\begin{tabular}{lccc} 
Group & $\begin{array}{c}\text { Age at EEG recording } \\
\text { (mean years } \pm \text { SD) }\end{array}$ & $\begin{array}{c}\text { Amplification experience 1st Cl } \\
\text { (mean years } \pm S D)\end{array}$ & $\begin{array}{c}\text { Amplification experience 2nd Cl } \\
\text { (mean years } \pm S D)\end{array}$ \\
CTRL & $7.67 \pm 4.5$ & & \\
UCl & $4.9 \pm 2.15$ & $1.73 \pm 0.85$ & $0.31 \pm 0.16$ \\
$\mathrm{BCl}$ & $5.42 \pm 1.92$ & $2.73 \pm 1.12$ & 0.12 \\
\hline
\end{tabular}

Table II. Paediatric clinical data.

\begin{tabular}{lcccccc} 
Participant & Gender & Atiology & Age at 1st Cl & Side 1st Cl & Age at 2nd Cl & Side 2nd Cl \\
1 & $\mathrm{M}$ & Unknown & 1.92 & $\mathrm{R}$ & $\mathrm{R}$ & 7.25 \\
2 & $\mathrm{~F}$ & Unknown & 7.58 & $\mathrm{~L}$ & 4.33 & $\mathrm{R}$ \\
3 & $\mathrm{~F}$ & Unknown & 4.17 & $\mathrm{R}$ & $\mathrm{L}$ \\
4 & $\mathrm{~F}$ & Unknown & 1.42 & $\mathrm{R}$ & 2.92 & $\mathrm{~L}$ \\
5 & $\mathrm{~F}$ & Unknown & 1.75 & $\mathrm{R}$ & $\mathrm{L}$ & $\mathrm{L}$ \\
\hline
\end{tabular}

by speech audiometry, simplified bisyllabic word lists by Turrini, ${ }^{32}$ ) for children $>5$ years and an "appropriate for age" score, assessed by the Sentence Comprehension Test in Italian ${ }^{33}$. All patients wore Nucleus ${ }^{\circledR}$ cochlear implants produced by Cochlear Ltd. (Cochlear Ltd, Sydney, Australia) and used ACE strategy for the encoding of the sound. All CI electrodes were active with normal impedance levels, 900 pps stimulation rate, and ADRO preprocessing algorithm. On the day that the EEG registration was performed, all the patients previously underwent warble-tone free-field audiometry and speech audiometry to ensure their hearing and speech recognition abilities were adequate. The EEG recordings were performed at IRCCS Fondazione Santa Lucia in according to the Declaration of Helsinki after receiving the approval by the local ethical committee. Informed consent was obtained from the parents of all experimental subjects.

Study participants underwent EEG recordings during an audio-video stimulation lasting 3 minutes, extracted from the cartoon Fantasia (Walt Disney, 1940) with the original music of D. Paradisi. This specific cartoon was chosen because it is characterised by a close association between music and images. Additionally, the cartoon nature of the stimulation is particularly suitable to engage the attention of young children, as it is the type of video usually watched by this population. In the selected video extracts, there were ostriches, dressed and dancing like classical dancers, dancing on the Paradisi's music. All the experimental groups performed the test sitting on a comfortable chair, placed at a distance of one meter from the screen used for the stimulation. The audio of the video was set so that it did not have intensity peaks greater than $65 \mathrm{~dB}$. In particular, three versions of the video clip were proposed during the EEG recordings: the first was composed of original audio and video and it will be called hereafter as "normal" movie (normal condition). The second version of the movie was obtained in the following way from the original normal movie: 1) the video was maintained unchanged; 2) the audio signal was played in reverse mode, from the last note to the first one, in order to generate an undecipherable sound but maintaining the same global acoustic pressure generated in the normal condition. The software Audacity was used to realise this stimulus, which will be called hereafter as "distorted". The third version of the movie was generated by presenting the same video than in the normal and distorted stimuli but without sound. In this case, the movie was called "mute". The three stimulations (normal, distorted, mute) were counterbalanced among subjects to avoid a sequence effect.

\section{Experimental design with adult subjects}

The adult population was composed of $7 \mathrm{NH}$ control subjects, 7 UCI and 6 BCI patients (Tables III and IV). Several of the adult patients had residual hearing in both ears before any implantation, as indicated in Table IV by the PTA calculated as the average threshold among 250 $\mathrm{Hz}, 500 \mathrm{~Hz}, 1 \mathrm{kHz}, 2 \mathrm{kHz}$ frequencies for each ear. As for the paediatric group, the UCI adult sample was also composed of poor contralateral hearing aid users. Five of 7 adult patients enrolled in the study received speech therapy for at least 1 yeast before the testing, two of whom did not undergo speech therapy. All patients wore coch- 
Table III. Adult groups age and time of cochlear implant experience.

\begin{tabular}{|c|c|c|c|}
\hline Group & $\begin{array}{l}\text { Age at EEG recording } \\
\text { (mean years } \pm S D \text { ) }\end{array}$ & $\begin{array}{l}\text { Amplification experience 1st Cl } \\
\text { (mean years } \pm \text { SD) }\end{array}$ & $\begin{array}{c}\text { Amplification experience } 2 \mathrm{nd} \mathrm{Cl} \\
\text { (mean years } \pm \mathrm{SD} \text { ) }\end{array}$ \\
\hline CTRL & $37.57 \pm 14.55$ & & \\
\hline $\mathrm{UCl}$ & $48.87 \pm 14.74$ & $2.89 \pm 3.51$ & \\
\hline $\mathrm{BCl}$ & $49.71 \pm 15.82$ & $3.87 \pm 3.55$ & $0.65 \pm 0.35$ \\
\hline
\end{tabular}

Table IV. Adult clinical data (PTA calculated as the average threshold among $250 \mathrm{~Hz}, 500 \mathrm{~Hz}, 1 \mathrm{KHz}, 2 \mathrm{KHz}$ frequencies for each ear).

\begin{tabular}{|c|c|c|c|c|c|c|c|c|c|c|}
\hline Participant & Gender & Deafness & $\begin{array}{l}\text { Age at 1st } \\
\mathrm{Cl}(\mathrm{mo})\end{array}$ & $\begin{array}{l}\text { PTA Pre-1st } \\
\text { CI Right side }\end{array}$ & $\begin{array}{l}\text { PTA Pre- } \\
\text { 1st CI Left } \\
\text { side }\end{array}$ & $\begin{array}{l}\text { Side of } \\
\text { 1st Cl }\end{array}$ & $\begin{array}{l}\text { Age at 2nd } \\
\mathrm{Cl}(\mathrm{mo})\end{array}$ & $\begin{array}{l}\text { PTA Pre-2nd } \\
\text { CI Right side }\end{array}$ & $\begin{array}{l}\text { PTA Pre-2nd } \\
\text { CI Left side }\end{array}$ & $\begin{array}{l}\text { Side of } \\
\text { 2nd Cl }\end{array}$ \\
\hline 1 & $M$ & Postverbal & 61.08 & 62.5 & 95 & $\mathrm{R}$ & 61.33 & 88.75 & 95 & L \\
\hline 2 & $F$ & Periverbal & 52.08 & 95 & 95 & $L$ & 54.33 & 95 & 108.33 & R \\
\hline 3 & $M$ & Postverbal & 32.67 & 81.67 & 120 & $\mathrm{R}$ & 34.58 & 100 & 120 & $L$ \\
\hline 4 & $M$ & Postverbal & 47.08 & 57.5 & 91.25 & L & & 57.5 & 103.75 & \\
\hline 5 & $\mathrm{~F}$ & Periverbal & 20.58 & 95 & 95 & $\mathrm{R}$ & 25.42 & 120 & 102.5 & $L$ \\
\hline 6 & $M$ & Periverbal & 41.00 & 101.25 & 87.5 & $\mathrm{R}$ & 50.50 & 115 & 97.5 & $L$ \\
\hline 7 & $M$ & Postverbal & 67.33 & 80 & 120 & $\mathrm{~L}$ & 68.08 & 120 & 120 & $\mathrm{R}$ \\
\hline
\end{tabular}

lear implants produced by Cochlear Ltd. (Cochlear Ltd, Sydney, Australia), used ACE strategy for the encoding of the sound, and all CI electrodes were active with normal impedance levels, 900 pps stimulation rate and ADRO preprocessing algorithm. On the day that the EEG registration was performed, all patients previously underwent warble-tone free-field audiometry and speech audiometry to ensure their hearing and speech recognition abilities were adequate. The EEG recordings were performed at IRCCS Fondazione Santa Lucia in according to the Declaration of Helsinki after receiving the approval by the local ethics committee. Informed consent was obtained from all participants.

For the adult population, the visual stimuli consisted of a 3-minute-length piece from the musical West Side Story. The extract of the musical was about a very animated scene of a sort of dance challenge, with many dancers and a strongly rhythmical music. In particular, in the video clip there is no speech, but only music and dance strictly related. As for the paediatric population, three versions of the video clip were watched by each participant and the three movies were named according to the labeling already described in the previous section (normal, distorted and mute).

\section{Behavioural rating}

Adult subjects were asked to rate how much they liked or disliked each condition (normal, distorted and mute) on a scale ranging from 0 (lowest) to 10 (highest) immediately after watching the video. This self-report inves- tigation was conducted only on adult subjects because children were not able to express on a number scale their like/dislike rating.

\section{EEG data recording and signal processing}

The EEG data were acquired using the BeMicro device (EBNeuro spa, Italy), equipped with a 16 electrode cap according to the 10-20 International System. In particular, the signal was gathered from the following locations: F7, F8, F3, Fz, F4, T7, C3, Cz, C4, T8, P3, Pz, P4, O1, O2. The signals were acquired maintaining the value of the impedance below the $10 \mathrm{k} \Omega$ and at a sampling frequency of $256 \mathrm{~Hz}$. This EEG activity at rest (in the present experiment corresponding to the open eyes condition looking at a black screen without any stimuli played) was used to calculate the Individual Alpha Frequency (IAF) to accordingly define individual alpha and band ranges of the EEG spectrum ${ }^{34}$.

The pre-processing of the EEG data was performed using the EEGLAB software ${ }^{35}$ according to the following steps. The first was to perform a band-pass filtering of the EEG data in the range 2-30 Hz, in order to exclude most muscular artifacts (beyond $30 \mathrm{~Hz}$ ) and the drift due to the changing of the contact impedance (below $2 \mathrm{~Hz}$ ). Successively, the independent component analysis (ICA) was performed to remove signal artifacts mainly due to eye movements. Therefore, components related to eye-blinks were first detected and then removed. The EEG trace was segmented into trials lasting $1 \mathrm{sec}$, thus obtaining 180 tri- 
als for each subject and for each movie condition analysed. EEG spectral analysis was performed by calculating the power spectral density ${ }^{36}$ (PSD) of the acquired signals during the different conditions with a frequency resolution of $0.3 \mathrm{~Hz}$. The EEG PSD values obtained were averaged within the alpha frequency band whose range was calculated in accordance with the definition of the $\mathrm{IAF}^{34}$, i.e. alpha range is between [IAF-4, IAF+2].

In order to analyse the EEG activity when the novelty effect of the stimulation was ended, the following analyses were conducted considering the time interval corresponding to the second minute of stimulation (from trial 61 to 120).

\section{PSD frontal imbalance index}

The use of the approach/withdrawal theory has been validated over the last 20 years by a large number of studies since its formulation in the early $1990 \mathrm{~s}^{37-41}$. In the present study, the imbalance of the EEG spectral activity in the alpha frequency band over the prefrontal areas has been chosen as the main index for the evaluation of approach/ withdrawal towards the stimuli. This index was then estimated for each subject and for each condition analysed. In particular, the approach/withdrawal (AW) index is defined as follows:

$\mathrm{AW}=\mathrm{PSDR}-\mathrm{PSDL}$

being the PSDR the mean value of PSD calculated on the frontal right electrodes F8, F4, and the PSDL the average value of PSD related to frontal electrodes F7, F3. The AW index was calculated in each population for each movie condition. Positive values would indicate relative greater right alpha frontal activity, so suggesting an approach tendency; vice versa negative values would underline relative higher left alpha frontal activity, suggesting a withdrawal tendency.

\section{Partial directed coherence}

The estimation of the functional connectivity by means of partial directed coherence (PDC) ${ }^{42}$ allows to analyse the causal relationships between the EEG signals acquired from the different electrodes during the execution of a task. PDC is a frequency domain representation that allows the inference of functional relationships between electrodes, and so between localised cortical areas. Once estimated the connectivity between the EEG signal gathered from the different electrodes, it was necessary to apply a statistical validation method to distinguish the real connections from those arising due to random fluctuations and measurement errors. The value of effective connectivity for a given pair of electrodes, obtained by computing PDC $^{4344}$, must be statistically compared with a threshold level which is related to the lack of transmission between considered regions of interest (ROIs) (null hypothesis). Threshold values were estimated using asymptotic statistic $^{45} 46$. Details of the applied methodology have been provided elsewhere ${ }^{47} 48$. After the validation process, the PDC estimation is averaged within the alpha band defined according to the $\mathrm{IAF}^{34}$ to take into account the variability among subjects of the alpha peak in the spectrum. Specifically, we defined the alpha band ranging between [IAF-4, $\mathrm{IAF}+2]$.

\section{Graph theory}

A graph consists of a set of vertices (or nodes) and a set of edges (or connections) indicating the presence of some sort of interaction between the vertices. The PDC previously obtained in the present study was selected as the current adjacency matrix. The adjacency matrix contained the information about the connectivity structure of the graph. In graph theory, a path or a walk is a sequence of vertices, in which from each of its vertices, there is a connection to the next vertex in the sequence. Such adjacency matrix can be used for the extraction of salient information about the characteristic of the investigated network, by defining several indexes based on the elements of such matrix.

In order to obtain a characterisation of the global cerebral networks via graph theory, we used the following indices already present in literature ${ }^{49}$ : degree and local efficiency. Degree: a measure of "centrality", it is calculated as the number of links connected to a node, somehow a measure of the "importance" of a node in a network Local efficiency: a measure of the "segregation" of the network, related to the shortest path between two nodes both neighbours of the node object of measurement of its local efficiency and it is calculated as the average of the local efficiency of all nodes belonging to the network ${ }^{50}$.

In addition, we defined the following two new ones to obtain a specific characterization of the frontal asymmetries, analogously to the AW index. In particular, we defined the imbalance of out degree as the ratio between the differences of the out degrees (i.e. outgoing connections) of the two hemispheres of frontal areas and the total out degree computed across frontal nodes. Specifically:

\section{[IMB]_(Out Degree $)=([$ Out Degree $] R$ - [Out Degree]_L)/[Out Degree]_Tot}

where the out degreeR is the average degree among nodes in frontal right hemisphere, out degreeL is the average degree among nodes in frontal left hemisphere and out degreeTot is the total degree of the nodes across all frontal nodes. 
According to the formula, the minimum value that this index can assume is -1 . In this case, all the outgoing connections comes from the left hemisphere. Analogously, its maximum value is equal to 1 , and is representative of the opposite situation with all the outgoing connections coming from the right hemisphere. If both hemispheres have the same number of outgoing connections, this index is zero. Since the out degree index represents the number of links outgoing from a node, it would reflect the flow of information from a certain brain region, and finally a measure of cerebral activity in that area. In the just introduced index, the imbalance of out degree would shape the difference in outgoing flow information level between the right and left frontal areas.

The second index is the imbalance of local efficiency, which is defined as the ratio between the difference of the mean local efficiency computed among right and left frontal nodes and the local efficiency areas across all frontal nodes.

$[\mathrm{IMB}] \_(\text {Loc Eff })=\left([\right.$ loc eff $] \_\mathrm{R}-\left[\right.$ loc eff] $\left.\_L\right) /[$ loc eff $] \_$Tot

where:

loc eff_ $\mathrm{R}$ is the mean local efficiency across nodes of the frontal right hemisphere, loc eff_L is the mean local efficiency across nodes of the frontal left hemisphere, whereas the loc eff_Tot represents the mean local efficiency across all frontal nodes. The imbalance index of local efficiency ranges between $[-1 ; 1]$ and, depending on the prevailing hemisphere, it can reach -1 (only the left hemisphere contributes) or +1 (only the right hemisphere contributes). When the contribution of the two hemispheres is balanced this index is zero. The local efficiency reflects the tendency of a network to create subgroups of elements (clusters) strongly related among them, probably suggesting a sort of specialisation within the network. This index constitutes a measure of efficiency, so the introduced imbalance of local efficiency index would show the difference in efficiency between the right and left frontal areas. The indices were calculated for all subjects and conditions. Further results also showed an interesting pattern in the evolution of functional network complexity: networks were relatively closer to random in the youngest and the oldest groups, and relatively more 'small-world' like in the intermediate age group ${ }^{51}$. This suggests that network evolution during development may be characterised by the gradual acquisition of order in random networks, converting them to optimal 'small-world' networks. A study in children, who had EEG recorded at age five and age seven, supports this hypothesis on brain network development ${ }^{52}$.

\section{Statistical analysis}

For each experimental group (CTRL, UCI, BCI) and cerebral index (AW, IMB out degree, IMB loc eff) a repeated measures ANOVA was carried out, in which the dependent variables were represented by the indices employed to summarise the estimated brain network, and the independent factor by the movie conditions (normal, distorted and mute). The statistical analysis was completed by performing the post-hoc Duncan test at the 0.05 level of significance.

\section{Results}

\section{Behavioural rating}

Concerning the rating of the different conditions within each experimental group, only cochlear implant patients showed a significant difference between conditions (UCI: $p=0.03, F=4.8$; BCI: $p<0.001, F=15.07$; Control: $\mathrm{p}=0.09, \mathrm{~F}=2.96$ ) (Fig.1).

The comparison between normal, distorted and mute conditions in UCI and BCI subjects revealed a significant difference between normal and distorted conditions ( $p=0.023$ and $p=0.002$ respectively) and between normal and mute conditions $(\mathrm{p}=0.021$ and $\mathrm{p}<0.001$ respectively). The Control group, although without reaching statistical significance, showed absolute values with a trend similar to the one reported in UCI and BCI. In both UCI and BCI groups, the comparison between distorted and mute conditions did not reach statistical significance. However, the mute condition for both the UCI and $\mathrm{BCI}$ groups showed the lowest ratings, while the Control group showed the lowest value for the distorted condition.

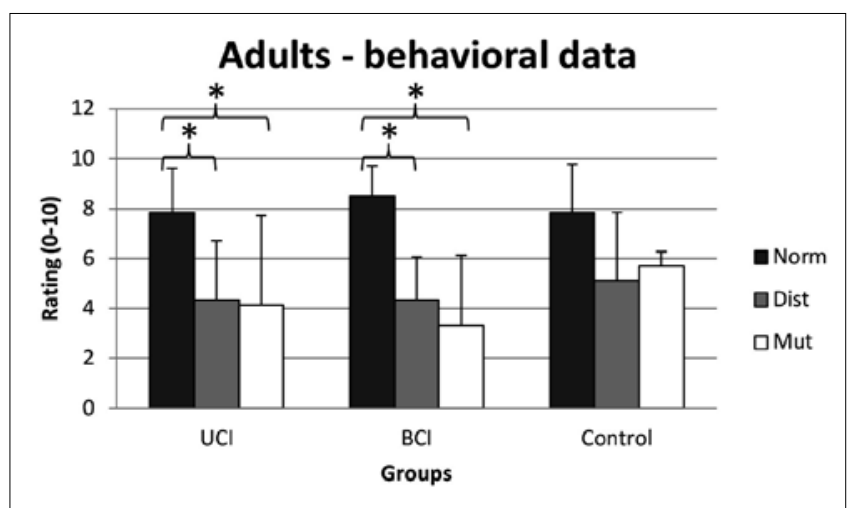

Fig. 1. Adult subjects rating of the different video versions (normal, distorted and mute) on a scale ranging from 0 (lowest pleasantness) to 10 (highest pleasantness). The comparison between normal, distorted and mute conditions in unilateral cochlear implant (UCI) and bilateral cochlear implant (BCl) subjects revealed a significant difference between normal and distorted conditions ( $p=0.023$ and $p=0.002$, respectively) and between normal and mute conditions ( $p=0.021$ and $p<0.001$, respectively). "Control" stands for normal hearing control subjects. 


\section{PSD alpha asymmetries}

In general, in both adults and children, a congruency among patterns between BCI patients and CTRL subjects was observed. In particular, both the BCI and CTRL groups showed lowest approach values for the distorted condition, in comparison to normal and mute ones. All paediatric groups, UCI, BCI and CTRL, reported the same pattern, characterised by lowest values for the distorted condition and highest values for the mute one. All adult groups showed a statistically significant difference between the normal and distorted condition and between distorted and mute, and UCI and BCI groups reported highest values for the normal condition. Concerning paediatric groups, only the CTRL group showed a significant difference in the above-cited comparisons, and the BCI group showed a significant difference only in the comparison between mute and the other conditions, while the UCI group did not show any significant difference between conditions.

\section{Unilateral group (UCI)}

Paediatric population. There was no significant difference between the experimental conditions $(\mathrm{p}=0.84, \mathrm{~F}=0.17)$ (Fig. 2a). It was possible to see a trend of the pattern shown by the Control group (Fig. 2e) (see below): lowest values for the distorted condition, then the normal and mute conditions progressively higher.

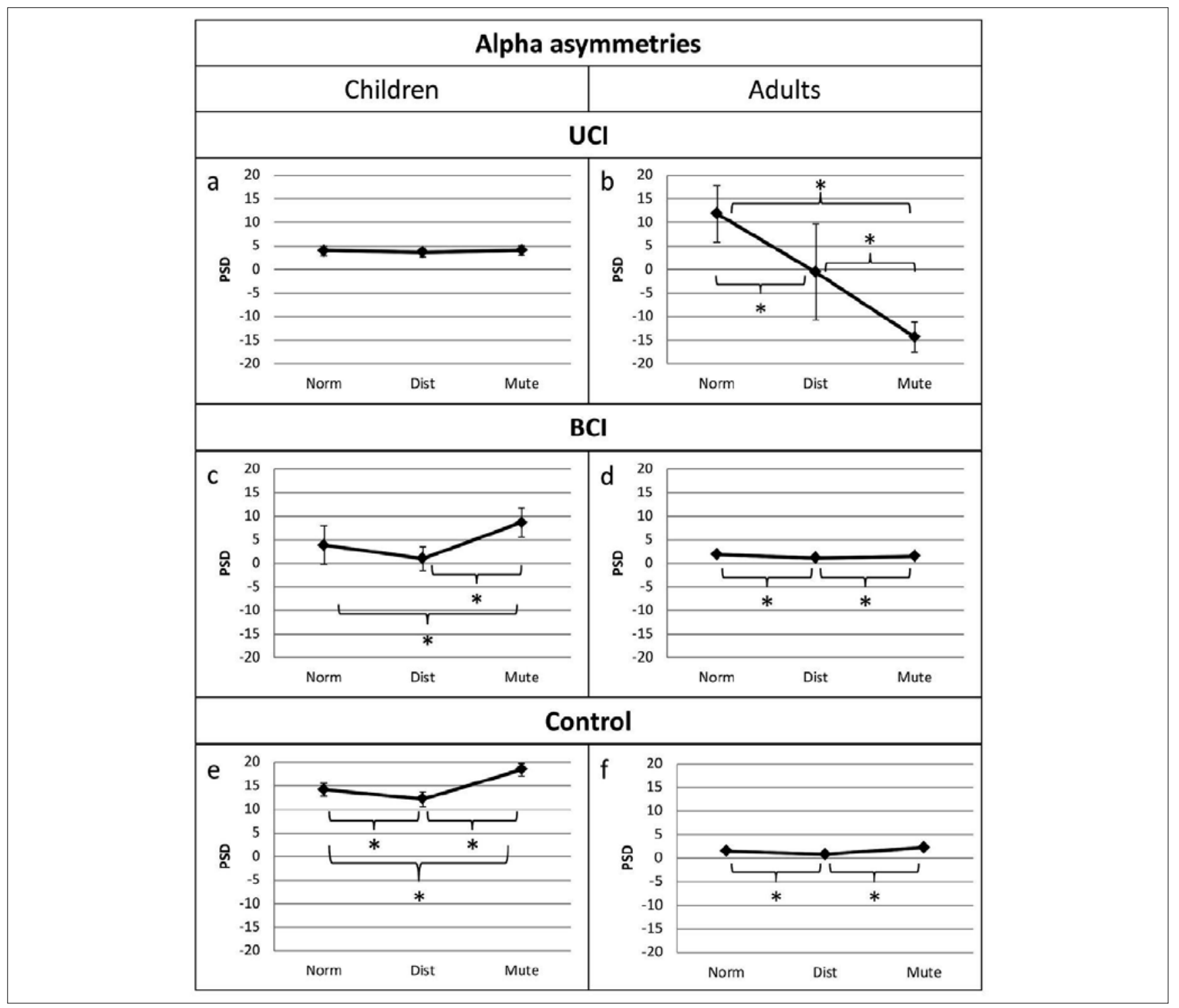

Fig. 2. EEG alpha asymmetries reported in the paediatric (left panels) and adult groups (right panels). Asterisks indicate statistically significant differences. Control = normal hearing control subjects; $\mathrm{UCl}=$ unilateral cochlear implant subjects; $\mathrm{BCl}=$ bilateral cochlear implant subjects. 
Adult population. There was a significant difference between the three movie conditions $(\mathrm{F}=12.94, \mathrm{p}<0.001)$ (Fig. 2b). The mute condition presented the lowest value, followed by the distorted and normal movies, and the pairwise comparisons were all characterised by significant differences (Duncan's test, normal and mute $\mathrm{p}=0.004$; normal and distorted $\mathrm{p}=0.0449$; distorted and mute $\mathrm{p}<0.001$ ).

\section{Bilateral group (BCI)}

Paediatric population. There was a significant difference between the experimental movies $(\mathrm{p}<0.002, \mathrm{~F}=6.6)$. Furthermore, a significant difference was present in the comparison between normal and mute (Duncan's test $\mathrm{p}<0.05$ ) and distorted and mute (Duncan's test $\mathrm{p}<0.05$ ). The above cited pattern concerning frontal imbalance (distorted $\rightarrow$ normal $\rightarrow$ mute) was also seen in this group.

Adult population. There was a significant difference between all movies $(\mathrm{p}<0.001, \mathrm{~F}=28.31)$ (Fig. $2 \mathrm{~d})$. The pairwise comparisons also showed statistically significant differences between normal and distorted $\mathrm{p}=0.025$ ) and between mute and distorted $(\mathrm{p}=0.009)$.

\section{Control group}

Paediatric population. There was a significant difference between all the experimental conditions $(p=0.001$, $\mathrm{F}=19.3$ ) (Fig. 2e). In particular, concerning the distorted condition, the front imbalance index value was significantly lower than the normal and mute condition (Duncan's test, $p=0.049$ and $p<0.001$, respectively), and there was also a significant difference between the normal and mute conditions $(\mathrm{p}<0.001)$.

Adult population. A significant difference was seen between all types of movies ( $p=0.0311, F=4.16$ ) (Fig. 2f). Additionally, the distorted movie presented a frontal imbalance index that was statistically significantly lower than normal (Duncan's test, $\mathrm{p}=0.0179$ ) and mute (Duncan's test, $\mathrm{p}=0.0424$ ) conditions.

\section{Graphs indices asymmetries}

The aforementioned connectivity indices were computed and compared for each population (paediatric, adult) and experimental condition. Below, we report the results related to the indices that were significantly modulated across populations and conditions: the imbalance of out degree and the imbalance of local efficiency.

Concerning the imbalance of out degree, the BCI and UCI groups showed the lowest values for the distorted condition, which was significantly lower for the distorted condition in comparison to normal except for the paediatric UCI group, where there was only a trend. Both UCI groups showed the highest values for the normal condition and lowest values for the mute video.

Concerning the imbalance of local efficiency, the BCI and CTRL groups showed the lowest values for the distorted condition. Furthermore, UCI children BCI adults and both CTRL groups had the highest values for the normal condition.

\section{Unilateral group (UCI)}

Paediatric population. The indices that showed a significant difference in the CTRL group were analysed in the UCI group. The imbalance of out degree index showed a significant difference $(\mathrm{p}=0.02, \mathrm{~F}=5.78)$ (Fig. 2a), but Duncan's post-hoc comparisons did not show significant differences, even if the comparison between the mute and the distorted conditions was just below the significance threshold $(p=0.057)$. The analysis of the imbalance of local efficiency index reached statistical significance $(\mathrm{p}=0.025, \mathrm{~F}=5.875)$ (Fig. $4 \mathrm{a})$, and the comparison between normal and mute $(p=0.045)$ and between distorted and mute $(\mathrm{p}=0.002)$ conditions were also significantly different.

Adult population. The indices that showed a significant difference in the CTRL group were analysed in the UCI group. Neither the imbalance of out degree index (Fig. 3b) $(p=0.07, F=3.29)$ nor the imbalance of local efficiency index (Fig. 4b) reached statistically significant differences $(\mathrm{p}=0.84, \mathrm{~F}=0.177)$.

\section{Bilateral group (BCI)}

Paediatric population. The indices that showed a significant difference in the CTRL group were analysed in the BCI group. The imbalance of out degree index analysis showed a trend similar to the that present in CTRL subjects $(\mathrm{p}=0.89, \mathrm{~F}=0.46)(\mathrm{Fig} .3 \mathrm{c})$, characterised by distorted condition values lower than mute and normal conditions; similarly the imbalance of local efficiency index analysis did not reveal any significant difference $(\mathrm{p}=0.478, \mathrm{~F}=0.857$ ) (Fig. 4c).

Adult population. The indices that showed a significant difference in the CTRL group were analysed in the BCI group. Both the imbalance of output degree index $(\mathrm{p}=0.024, \mathrm{~F}=5.58)$ (Fig. 3) and the imbalance of local efficiency index ( $\mathrm{p}=0.046, \mathrm{~F}=4.64)$ (Fig. 4d) showed a significant difference. In the imbalance of local efficiency index, the distorted condition value was significantly lower than the normal and mute conditions (Duncan's test, $p=0.038$ and $p=0.022$, respectively). Concerning the imbalance of out degree index, similar results were obtained. The distorted condition value was significantly lower than that in the normal and mute conditions $(\mathrm{p}=0.025$ and 


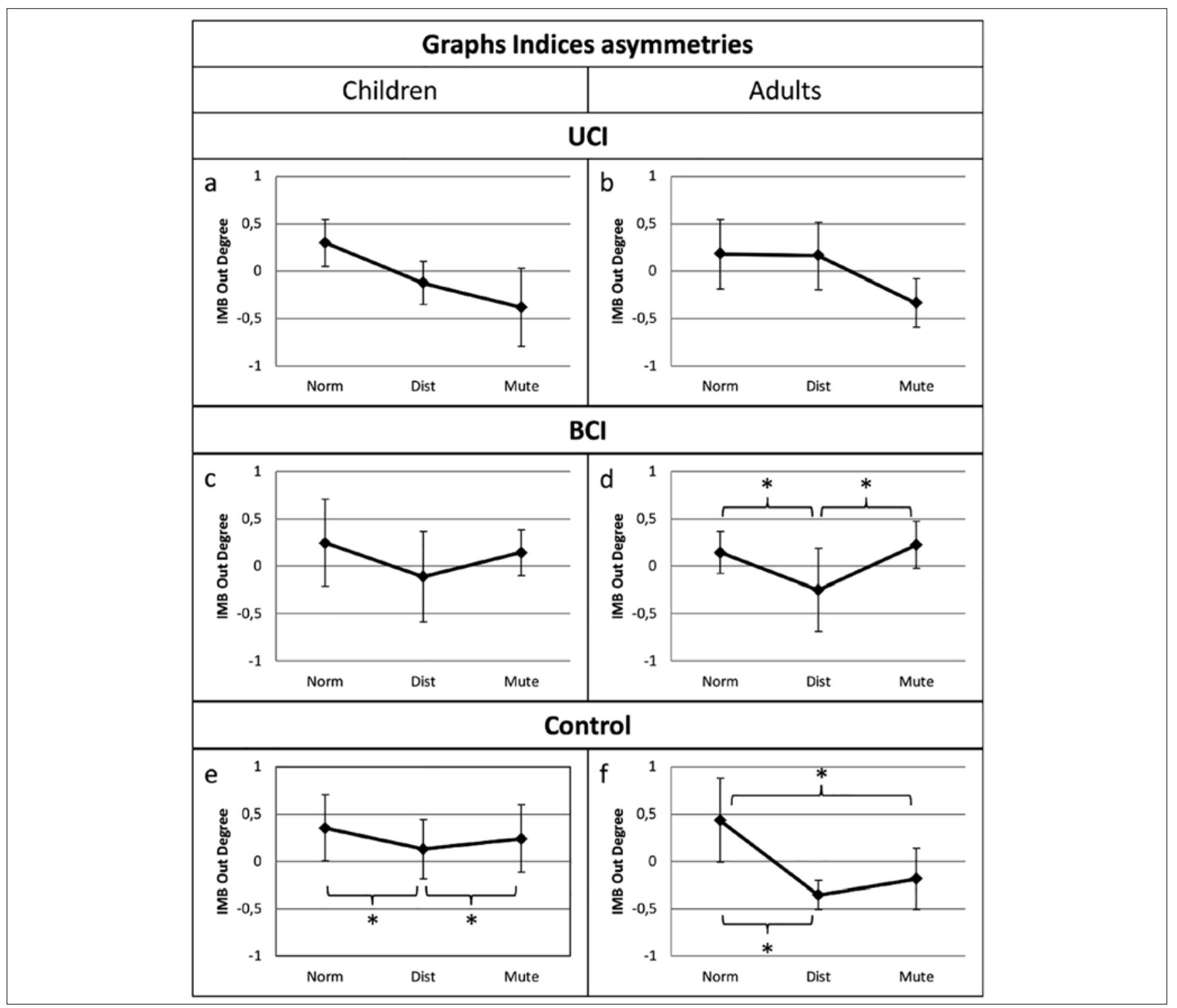

Fig. 3. EEG imbalance of out degree graph index reported in the paediatric (left panels) and adult groups (right panels). Asterisks indicate statistically significant differences. Control = normal hearing control subjects; $\mathrm{UCl}=$ unilateral cochlear implant subjects; $\mathrm{BCl}=$ bilateral cochlear implant subjects.

$\mathrm{p}=0.017)$. Finally, the trend resulting in the imbalance of out degree index was similar to that observed in CTRL subjects.

\section{Control group}

Paediatric population. The analysis of the imbalance of the out degree showed a significant difference $(\mathrm{p}=0.02$, $\mathrm{F}=6.64)$, showing higher activity in the normal and mute conditions with respect to the distorted condition ( $p=0.005$ and $p=0.016$, respectively) (Fig. 3e).

A significant difference was seen in the imbalance of local efficiency index analysis ( $\mathrm{p}=0.03, \mathrm{~F}=5.6)(\mathrm{Fig} .4 \mathrm{e})$. Duncan's post-hoc test highlighted a significant reduction in the distorted condition, which was characterised by a negative value vs. the positive values of the normal and mute conditions ( $\mathrm{p}=0.016$ and $\mathrm{p}=0.025$ ).

No significant differences were seen in the analysis of: the global efficiency index $(\mathrm{p}=0.89, \mathrm{~F}=0.11)$, local efficiency index $(\mathrm{p}=0.87, \mathrm{~F}=0.14)$, asymmetry between hemispheres index $(\mathrm{p}=0.58, \mathrm{~F}=0.59)$, degree index $(\mathrm{p}=0.63$, $\mathrm{F}=0.48)$, input degree index $(\mathrm{p}=0.61, \mathrm{~F}=0.48)$, out degree index $(\mathrm{p}=0.61, \mathrm{~F}=0.49)$, imbalance degree index ( $\mathrm{p}=0.43, \mathrm{~F}=0.91)$ or imbalance of input grade index $(\mathrm{p}=0.65, \mathrm{~F}=0.45)$.

Adult population. The imbalance of the out degree index analysis showed a significant difference $(p=0.0144$, 


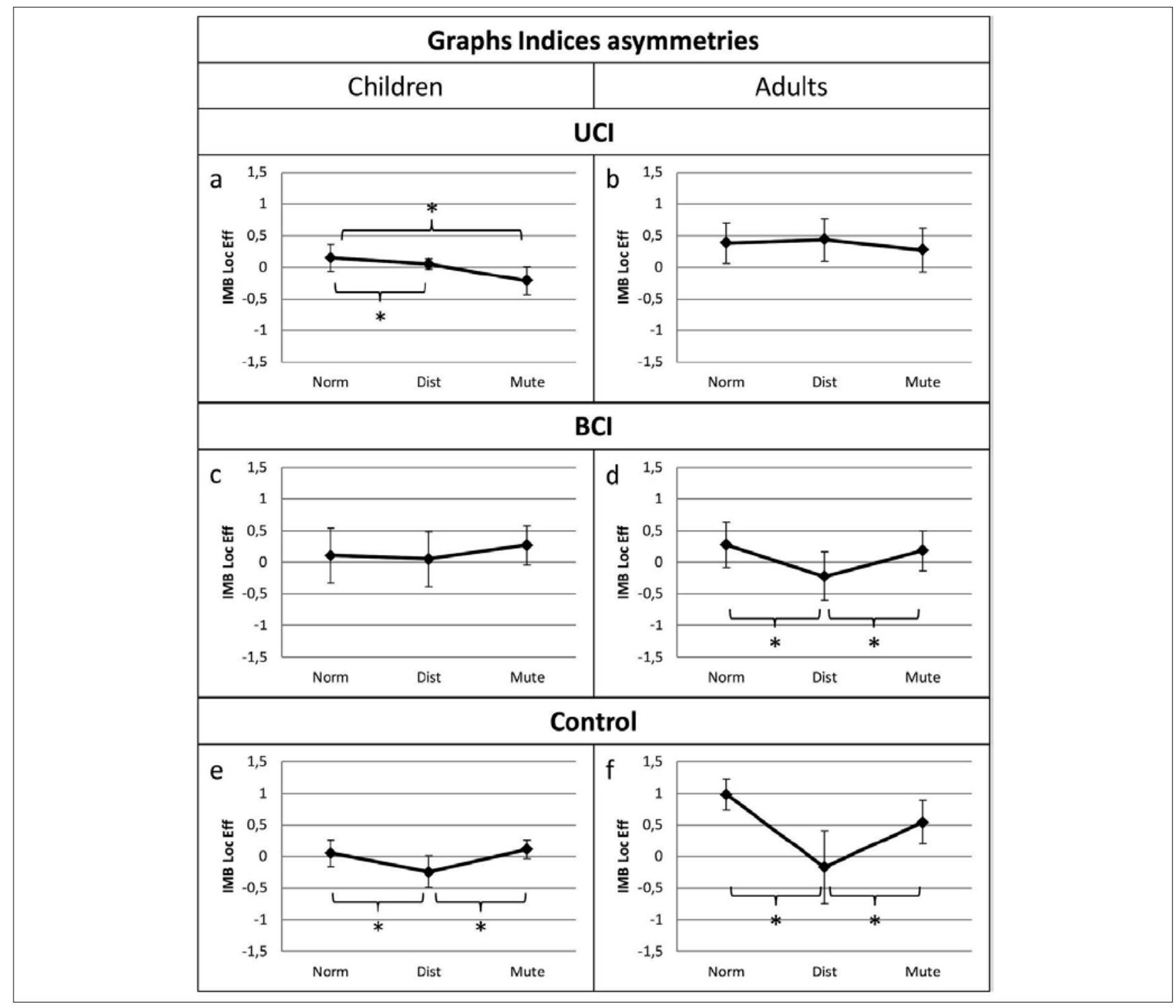

Fig. 4. EEG imbalance of local efficiency graph index reported in the paediatric (left panels) and adult groups (right panels). Asterisks indicate statistically significant differences. Control = normal hearing control subjects; $\mathrm{UCl}=$ unilateral cochlear implant subjects; $\mathrm{BCl}=$ bilateral cochlear implant subjects .

$\mathrm{F}=4.25$ ) with the Normal condition expressing a higher value in comparison to the Distorted and Mute conditions $(\mathrm{p}=0.001$ and $\mathrm{p}=0.0289$, respectively) (Fig. $3 \mathrm{f})$.

The analysis of the imbalance of local efficiency index showed a significant difference $(\mathrm{p}=0.0284, \mathrm{~F}=7.49)$, and the post-hoc comparisons revealed significantly lower values for the distorted condition in comparison to the normal and mute conditions ( $\mathrm{p}=0.0019$ and $\mathrm{p}=0.0449$, respectively) (Fig. 4f).

No statistically significant differences were found in the analysis of: the Global Efficiency index ( $p=0.763$, $\mathrm{F}=0.275)$; the Local Efficiency index $(\mathrm{p}=0.55$, $\mathrm{F}=0.622)$; Degree $(\mathrm{p}=0.553, \mathrm{~F}=0.586)$; the Input
Degree index $(\mathrm{p}=0.57, \mathrm{~F}=0.56)$; the Out Degree in$\operatorname{dex}(\mathrm{p}=0.55, \mathrm{~F}=0.58)$; the Imbalance Degree index $(\mathrm{p}=0.925, \mathrm{~F}=0.078)$; the Imbalance of Input Degree index $(\mathrm{p}=0.977, \mathrm{~F}=0.023)$.

\section{Discussion}

\section{PSD alpha asymmetries}

The most evident result was represented by the pattern of activation characterising the CTRL and BCI groups in both the adult and paediatric populations. This pattern showed the lowest values of the approach-withdrawal 
index, sign of less approach, for the distorted condition, in accordance with previous studies ${ }^{24}$. This suggests a higher capacity of discrimination and approach motivation towards normal music in the CTRL and BCI groups, in particular in the adult population, due to the similarity in music perception for these two groups, but not for the UCI group. Previous studies support this hypothesis, providing evidence of a higher capability of enjoyment of music by BCI vs. UCI subjects. In particular, subjects who underwent sequentially bilateral cochlear implantation reported that music generally sounded better: $90 \%$ reported it was more natural and $85 \%$ reported it was more pleasant after the second $\mathrm{CI}^{53}$. Additionally, it is interesting to note that, regarding CTRL and BCI patients, the adult population reported the highest alpha PSD (or approach) values for the normal condition. This could be explained by the fact that adult subjects usually have a past of some type of hearing, especially in the low frequency ranges. It has been hypothesised that access to early acoustical hearing in the lower frequency ranges appears to establish a basis for music perception, which can be accessed with later electrical $\mathrm{CI}$ hearing ${ }^{17}$. In addition, it has been shown that low-frequency acoustic hearing improves pitch discrimination performances in comparison to electric-only stimulation in CI adult users ${ }^{11}$. This cueing strategy relying on low frequencies would of course be denied to children selected for cochlear implantation because of the frequent absence of residual hearing in these patients. Moreover, also EEG show the presence of residual neural skills for music processing in adult CI users. These enable patients to automatically process changes in sound features in a musical context, except for automatic rhythm discrimination ${ }^{54}$.

In the literature, there is some evidence of the major interest and relying on visual stimuli than on auditory stimuli expressed by CI children ${ }^{55-57}$. In the present study, in contrast to expectations, $\mathrm{NH}$ and not only $\mathrm{CI}$ children groups also presented the highest values for the mute condition. This result could be explained by the observation that, in general, values obtained in CTRL subjects were lower than BCI or UCI subjects, probably suggesting a higher approach of all sensory modalities involved in CI groups. Furthermore, BCI children reported a value very close to zero for the distorted condition suggesting a not welldefined approach or withdrawal motivation toward the distorted movie, but higher and positive values for the normal and mute conditions. These results could suggest a deficit in the discrimination of "alternative versions" of music, as previously reported ${ }^{17}$, thus explaining a lacking response of interest or disinterest. Concerning UCI children, the patterns of the approach-withdrawal index were in general flatter than $\mathrm{NH}$ and BCI children, and did not show significant differences between conditions, supporting the hypothesis of the insufficient amplification for music perception provided by only one implant in these types of patients ${ }^{26}$. Furthermore, this evidence could be linked to the sensitive period that is necessary for correct establishment of auditory-visual integration in CI children ${ }^{58}$.

Finally, it is interesting to note the unique pattern displayed by the adult UCI group, not presenting a "V shape" but a "flat shape", characterised by a zero value for the distorted condition and negative values for the mute condition. The first observation could be explained by a deficit in discriminating the distorted condition due to insufficient amplification in the UCI group. The second observation could be mirrored by the behavioural rating data, reporting lowest values for the mute condition in cochlear implanted patients; finally, this scenario could reflect the sensation of "fear" that is incidentally and behaviourally self-reported by UCI subjects. This feeling shapes the neural correlates of withdrawal suggested by negative values.

\section{Graph indices asymmetries}

The results regarding the CTRL and BCI groups presented lowest values in the distorted condition, similarly to the frontal imbalance analysis for the same groups. Concerning the BCI adult group, there was a consistency in the pattern expressed in the two graph indices, and in the frontal asymmetry index, suggesting accordance and reliability between the first ones and among all indices. Concerning the UCI groups, in both the indices, they reported the same pattern characterised by lowest values for the mute condition (Fig. 4), both in children and the adult population. As discussed above, that could be linked to the "fearful" reaction of subjects to the absence of sound self-reported by patients. This aversive reaction could be expressed by UCI subjects and not by BCI subjects because of a more demanding and distressful approach to sound as a baseline condition in UCI patients, in whom the amplification provided may not be sufficient. An indirect proof of this hypothesis is that normal-like audiovisual segregation is possible in highly skilled cochlear implant users ${ }^{59}$, so that UCI individuals cannot rely on the same set of audio and visual cues, finally producing withdrawal in UCI in the mute condition.

Finally, considering the graph indices in the normal condition, it is interesting to note that positive values were ubiquitous in all the experimental groups and reaching significance in BCI and CTRL groups, showing a relative greater activity in the right frontal hemisphere. This con- 
sideration shows that the normal condition elicited positive graph index values and therefore approach behaviour, as suggested by brain functionality in the frontal areas. This brain functionality would reflect the normal hearing condition and the analogous condition restored by neuroplasticity in the BCI groups, but not in the UCI groups, even if a tendency toward the pattern is suggested even in unilateral implantation. As suggested by the graph indices (both the out degree and the local efficiency), similar considerations but in an opposite direction can be made for the neural activity underlying the reaction to the distorted condition, characterised by a relatively greater activity in the left frontal hemisphere and therefore a propensity to withdraw from the stimulus by CTRL and (mainly) BCI groups. Evidence of an alteration in connectivity has been already identified in neurological disorders, such as Alzheimer's disease, multiple sclerosis, traumatic brain injury and epilepsy, challenging the classical concept of neurological disorders producing either "local" or "global" efficiency alterations, and pointing to the overload and failure of hubs as a possible final common pathway in neurological disorders ${ }^{28}$.

Abstracting the EEG functional activity from the direction of the connection among nodes, and generalising to the level of synchronisation activity among them, the reaction to the listening of music pieces has been investigated. In particular, listening to pleasant music (by Bach and Mahler) characterised by melodic features following expected rules produced an increase in the left cognitive area activity; on the other hand, exposure to unpleasant music lacking predictable melodic features produced an higher right frontopolar activity ${ }^{60}$. These data could support the hypothesis that, as indexed by the imbalance of out degree and the imbalance of local efficiency in our study, the almost ubiquitous higher right alpha relative functional connectivity in response to normal music would underlie the catching of its predictive melodic features, while the higher left relative functional connectivity in response to the distorted condition mainly expressed by BCI and CTRL groups may underlie the catching of the novel/unpredictable nature of that musical version. The last statement, again, would imply an insufficient melodic cue detection by UCI subjects, so to enable them of the unpredictable nature of the distorted condition.

The sum of these evidences supports the hypothesis that the connectivity study is an alternative suitable approach for assessing approach tendency towards music in adults and children, although the clinical implications of altered connectivity pattern must be further explored.

\section{Conclusions}

Responses in the BCI groups were more similar to those shown by $\mathrm{NH}$ groups, thus supporting the evidence for lack of information provided by one cochlear implant. All indices investigated, frontal alpha band asymmetry and graphs (connectivity) indices reported accordance among results, suggesting the worthiness of also applying this type of analysis to approach/withdrawal motivation studies.

\section{Acknowledgements}

The authors deeply thank all the participants in the study. The EEG recordings were performed at IRCCS Fondazione Santa Lucia in according to the Declaration of Helsinki after receiving the approval by the local ethics committee. Informed consent was obtained from of all the experimental subjects or from the parents of the participants in case of children. The authors declare that they have no conflict of interests regarding the publication of this paper.

The present study was partially funded by Cochlear Research \& Development Ltd, UK.

\section{References}

1 Zatorre RJ, Salimpoor VN. From perception to pleasure: Music and its neural substrates. Proc Natl Acad Sci USA 2013;110:10430-7.

2 Koelsch S. Brain correlates of music-evoked emotions. Nat Rev Neurosci 2014;15:170-80.

3 Sladen DP, Zappler A. Older and younger adult cochlear implant users: speech recognition in quiet and noise, quality of life, and music perception. Am J Audiol 2015;24:31-9.

4 Chen JM, Amoodi H, Mittmann N. Cost-utility analysis of bilateral cochlear implantation in adults: a health economic assessment from the perspective of a publicly funded program. Laryngoscope 2014;124:1452-8.

5 Drennan WR, Rubinstein JT. Music perception in cochlear implant users and its relationship with psychophysical capabilities. J Rehabil Res Dev 2008;45:779-89.

6 Driscoll VD. The effects of training on recognition of musical instruments by adults with cochlear implants. Semin Hear 2012;33:410-8.

7 Torppa R, Huotilainen M, Leminen M, et al. Interplay between singing and cortical processing of music: a longitudinal study in children with cochlear implants. Front Psychol 2014;5:1389.

8 Rochette F, Moussard A, Bigand E. Music lessons improve auditory perceptual and cognitive performance in deaf children. Front Hum Neurosci 2014;8:488.

9 Limb CJ, Roy AT. Technological, biological, and acoustical 
constraints to music perception in cochlear implant users. Hear Res 2014;308:13-26.

10 Limb CJ. Cochlear implant-mediated perception of music. Curr Opin Otolaryngol Head Neck Surg 2006;14:337-40.

11 Gfeller K, Turner C, Oleson J, et al. Accuracy of cochlear implant recipients on pitch perception, melody recognition, and speech reception in noise. Ear Hear 2007;28:412-23.

12 McDermott HJ. Music perception with cochlear implants: a review. Trends Amplif 2004;8:49-82.

13 Green KM, Julyan PJ, Hastings DL, et al. Auditory cortical activation and speech perception in cochlear implant users: Effects of implant experience and duration of deafness. Hear Res 2005;205:184-92.

14 Limb CJ, Molloy AT, Jiradejvong P, et al. Auditory cortical activity during cochlear implant-mediated perception of spoken language, melody, and rhythm. J Assoc Res Otolaryngol 2009;11:133-43.

15 Ortmann M, Knief A, Deuster D, et al. Neural correlates of speech processing in prelingually deafened children and adolescents with cochlear implants. PLoS One 2013;8: e67696.

16 Naito Y, Tateya I, Fujiki N, et al. Increased cortical activation during hearing of speech in cochlear implant users. Hear Res 2000;143:139-46.

17 Hopyan T, Peretz I, Chan LP, et al. Children using cochlear implants capitalize on acoustical hearing for music perception. Front Psychol 2012;3:425.

18 Jung KH, Won JH, Drennan WR, et al. Psychoacoustic performance and music and speech perception in prelingually deafened children with cochlear implants. Audiol Neurootol 2012;17:189-97.

19 Vongpaisal T, Trehub SE, Schellenberg EG. Song recognition by children and adolescents with cochlear implants. J Speech Lang Hear Res 2006;49:1091-103.

20 Davidson RJ. Anxiety and affective style: role of prefrontal cortex and amygdala. Biol Psychol 2002;51:68-80.

21 Coan JA, Allen JJ. Frontal EEG asymmetry as a moderator and mediator of emotion. Biol Psychiatry 2004;67:7-49.

22 Schmidt LA, Trainor LJ. Frontal brain electrical activity (EEG) distinguishes valence and intensity of musical emotions. Cognition and Emotion 2001;15:487-500.

23 Maglione AG, Vecchiato G, Leone CA, et al. Different perception of musical stimuli in patients with monolateral and bilateral cochlear implants. Comput Mat Methods Med 2014;2014:e876290.

24 Marsella P, Scorpecci A, Vecchiato G, et al. Neuroelectrical imaging investigation of cortical activity during listening to music in prelingually deaf children with cochlear implants. Int J Pediatr Otorhinolaryngol 2014;78:737-43.

25 Vecchiato G, Maglione AG, Scorpecci A, et al. EEG frontal asymmetry related to pleasantness of music perception in healthy children and cochlear implanted users. Conf Proc IEEE Eng Med Biol Soc 2012;2012:4740-3.

26 Marsella P, Scorpecci A, Vecchiato G, et al. Neuroelectrical imaging study of music perception by children with unilat- eral and bilateral cochlear implants. Cochlear Implants Int 2014;15(Suppl1):S68-71.

27 Başar E, Başar-Eroglu C, Karakaş S, et al. Gamma, alpha, delta, and theta oscillations govern cognitive processes. Int $\mathrm{J}$ Psychophysiol 2001;39:241-8.

28 Stam CJ. Modern network science of neurological disorders. Nat Rev Neurosci 2014;15:683-95.

29 Bullmore E, Sporns O. Complex brain networks: graph theoretical analysis of structural and functional systems. Nat Rev Neurosci 2009;10:186-98.

$30 \mathrm{Wu}$ J, Zhang J, Ding X, et al. The effects of music on brain functional networks: a network analysis. Neuroscience 2013;250:49-59.

31 Daly I, Malik A, Hwang F, et al. Neural correlates of emotional responses to music: an EEG study. Neurosci Lett 2014;573:52-7.

32 Turrini M, Cutugno F, Maturi P, et al. Bisyllabic words for speech audiometry: a new Italian material. Acta Otorhinolaryngol Ital 1993;13:63-77.

33 Rustioni, D. Prove di valutazione della comprensione linguistica [The Linguistic Comprehension Test]. Florence, Italy: Organizzazioni Speciali; 1994.

34 Klimesch W. EEG alpha and theta oscillations reflect cognitive and memory performance: a review and analysis. Brain Res Brain Res Rev 1999;29:169-95.

35 Delorme A, Makeig S. EEGLAB: an open source toolbox for analysis of single-trial EEG dynamics including independent component analysis. J Neurosci Methods 2004;134:9-21.

36 Welch PD. The use of fast Fourier transform for the estimation of power spectra: A method based on time averaging over short, modified periodograms. IIEEE Trans Audio Electroac 1967;15:70-3.

37 Davidson RJ, Ekman P, Saron CD, et al. Approach-withdrawal and cerebral asymmetry: emotional expression and brain physiology. I J Pers Soc Psychol 1990;58:330-41.

38 Allen JJB, Kline JP. Frontal EEG asymmetry, emotion, and psychopathology: the first, and the next 25 years. Biol Psychol 2004;67:1-5.

39 Harmon-Jones E, Gable PA, Peterson CK. The role of asymmetric frontal cortical activity in emotion-related phenomena: a review and update. Biol Psychol 2010;84:451-62.

40 Price TF, Peterson CK, Harmon-Jones E. The emotive neuroscience of embodiment. Motiv Emot 2011;36:27-37.

41 Briesemeister BB, Tamm S, Heine A, et al. Approach the good, withdraw from the bad - a review on frontal alpha asymmetry measures applied psychological research. Psychology 2013;4:261-7.

42 Baccalá LA, Sameshima K. Partial directed coherence: a new concept in neural structure determination. Biol Cybern 2001;84:463-74.

43 Sameshima K, Baccalá LA. Using partial directed coherence to describe neuronal ensemble interactions. J Neurosci Methods 1999;94:93-103. 
44 Gourévitch B, Bouquin-Jeannès RL, Faucon G. Linear and nonlinear causality between signals: methods, examples and neurophysiological applications. Biol Cybern 2006;95:349-69.

45 Takahashi DY, Baccalá LA, Sameshima K. Connectivity inference between neural structures via partial directed coherence. J Appl Stat 2007;34:1259-73.

46 Schelter B, Winterhalder M, Eichler M, et al. Testing for directed influences among neural signals using partial directed coherence. J Neurosci Methods 2006;152:210-9.

47 Toppi J, Babiloni F, Vecchiato G, et al. Testing the asymptotic statistic for the assessment of the significance of partial directed coherence connectivity patterns. Conf Proc IEEE Eng Med Biol Soc 2011;2011:5016-9.

48 Vecchiato G, Toppi J, Maglione AG, et al. Neuroelectrical correlates of trustworthiness and dominance judgments related to the observation of political candidates. Comput Math Methods Med 2014;2014:434296.

49 Rubinov M, Sporns O. Complex network measures of brain connectivity: Uses and interpretations. Neuroimage 2010;52:1059-69.

50 Latora V, Marchiori M. Efficient behavior of small-world networks. Phys Rev Lett 2001;87:198701.

51 Smit DJA, Boersma M, van Beijsterveldt CEM, et al. Endophenotypes in a dynamically connected brain. Behav Genet 2010;40:167-77.

52 Boersma M, Smit DJA, Boomsma DI, et al. Growing trees in child brains: graph theoretical analysis of electroencephalography-derived minimum spanning tree in 5- and 7-year-old children reflects brain maturation. Brain Connect 2012;3:50-60.

53 Veekmans K, Ressel L, Mueller J, et al. Comparison of music perception in bilateral and unilateral cochlear implant users and normal-hearing subjects. Audiol Neurootol 2009; 14:315-26.

54 Timm L, Vuust P, Brattico E, et al. Residual neural processing of musical sound features in adult cochlear implant users. Front Hum Neurosci 2014;8:181.

55 Campbell J, Sharma A. Visual cross-modal re-organization in children with cochlear implants. PLoS One 2016;11:e0147793.

56 Harris J, Kamke MR. Electrophysiological evidence for altered visual, but not auditory, selective attention in adolescent cochlear implant users. Int J Pediatr Otorhinolaryngol 2014;78:1908-16.

57 Schorr EA, Fox NA, van Wassenhove V, et al. Auditoryvisual fusion in speech perception in children with cochlear implants. Proc Natl Acad Sci U S A 2005;102:18748-50.

58 Gilley PM, Sharma A, Mitchell TV, et al. The influence of a sensitive period for auditory-visual integration in children with cochlear implants. Restor Neurol Neurosci 2010;28:207-18.

59 Landry S, Bacon BA, Leybaert J, et al. Audiovisual segregation in cochlear implant users. PLoS One 2012;7.

60 Flores-Gutiérrez EO, Díaz J-L, Barrios FA, et al. Metabolic and electric brain patterns during pleasant and unpleasant emotions induced by music masterpieces. Int J Psychophysiol 2007;65:69-84.

Address for correspondence: Giulia Cartocci, Department of Molecular Medicine, Sapienza University of Rome; viale Regina Elena, 291, 00161 Rome, Italy. Tel. +39 06 49912223. E-mail: giulia.cartocci@uniroma1.it 